\title{
Impact of renal function on admission in COVID-19 patients: an analysis of the international HOPE COVID-19 (Health Outcome Predictive Evaluation for COVID 19) Registry
}

\author{
Aitor Uribarri ${ }^{1}$ (1) - Iván J. Núñez-Gil ${ }^{2} \cdot$ Alvaro Aparisi $^{1} \cdot$ Victor M. Becerra-Muñoz $^{3} \cdot$ Gisela Feltes $^{4}$. \\ Daniela Trabattoni ${ }^{5} \cdot$ Inmaculada Fernández-Rozas ${ }^{6} \cdot$ María C. Viana-Llamas $^{7} \cdot$ Martino Pepe $^{8}$. Enrico Cerrato ${ }^{9}$. \\ Thamar Capel-Astrua ${ }^{10}$ - Rodolfo Romero ${ }^{11}$ - Alex F. Castro-Mejía ${ }^{12}$ - Ibrahim El-Battrawy ${ }^{13,14}$. Javier López-País ${ }^{15}$. \\ Fabrizio D'Ascenzo ${ }^{16}$. Oscar Fabregat-Andres ${ }^{17}$. Alfredo Bardaji ${ }^{18} \cdot$ Sergio Raposeiras-Roubin ${ }^{19} \cdot$ Francisco Marín $^{20}$. \\ Antonio Fernández-Ortiz ${ }^{2}$. Carlos Macaya ${ }^{2}$. Vicente Estrada ${ }^{2}$ on behalf of HOPE COVID-19 Investigators
}

Received: 6 May 2020 / Accepted: 22 June 2020 / Published online: 29 June 2020

(c) Italian Society of Nephrology 2020

\begin{abstract}
Background Coronavirus disease 2019 (COVID-19) is a disease caused by severe acute respiratory syndrome coronavirus 2 (SARS-CoV-2). Despite its international aggressive extension, with a significant morbidity and mortality, the impact of renal function on its prognosis is uncertain.

Methods Analysis from the international HOPE-Registry (NCT04334291). The objective was to evaluate the association between kidney failure severity on admission with the mortality of patients with SARS-CoV-2 infection. Patients were categorized in 3 groups according to the estimated glomerular filtration rate on admission (eGFR $>60 \mathrm{~mL} / \mathrm{min} / 1.73 \mathrm{~m}^{2}$, eGFR $30-60 \mathrm{~mL} / \mathrm{min} / 1.73 \mathrm{~m}^{2}$ and eGFR $<30 \mathrm{~mL} / \mathrm{min} / 1.73 \mathrm{~m}^{2}$ ).

Results 758 patients were included: mean age was $66 \pm 18$ years, and $58.6 \%$ of patient were male. Only $8.5 \%$ of patients had a history of chronic kidney disease (CKD); however, $30 \%$ of patients had kidney dysfunction upon admission (eGFR $<60 \mathrm{~mL} /$ $\min / 1.73 \mathrm{~m}^{2}$ ). These patients received less frequently pharmacological treatment with hydroxychloroquine or antivirals and had a greater number of complications such as sepsis $(11.9 \%$ vs $26.4 \%$ vs $40.8 \%, \mathrm{p}<0.001)$ and respiratory failure $(35.4 \%$ vs $72.2 \%$ vs $62.0 \%, \mathrm{p}<0.001$ ) as well as a higher in-hospital mortality rate (eGFR $>60$ vs eGFR $30-60$ vs and eGFR $<30$, $18.4 \%$ vs $56.5 \%$ vs $65.5 \%, \mathrm{p}<0.001)$. In multivariate analysis: age, hypertension, renal function, $0^{2}$ saturation $<92 \%$ and lactate dehydrogenase elevation on admission independently predicted all-cause mortality.

Conclusions Renal failure on admission in patients with SARS-CoV-2 infection is frequent and is associated with a greater number of complications and in-hospital mortality. Our data comes from a multicenter registry and therefore does not allow to have a precise mortality risk assessment. More studies are needed to confirm these findings.
\end{abstract}

Keywords COVID-19 $\cdot$ Mortality $\cdot$ Registry $\cdot$ Prognosis $\cdot$ Acute kidney injury $\cdot$ Chronic kidney failure

\section{Abbreviations}

ACE2 Angiotensin-converting enzyme 2

AKI

Acute kidney injury

HOPE COVID-19 Investigators, Scientific Committee and collaborators are listed in Supplementary appendix.

Electronic supplementary material The online version of this article (https://doi.org/10.1007/s40620-020-00790-5) contains supplementary material, which is available to authorized users.

Aitor Uribarri

auribarrig@gmail.com

Extended author information available on the last page of the article
CKD Chronic kidney disease

COVID-19 Coronavirus disease 2019

eGFR Estimated glomerular filtration rate

RAAS Renin-angiotensin-aldosterone system

SARS-CoV-2 Severe acute respiratory syndrome coronavirus 2

SIRS Systemic inflammatory response syndrome 


\section{Introduction}

In January, 2020, a novel virus, severe acute respiratory syndrome coronavirus 2 (SARS-CoV-2), was identified as the causative agent for a cluster of pneumonia cases initially detected in Wuhan City, Hubei province, China [1]. SARS-CoV-2, which causes the disease now named coronavirus disease 2019 (COVID-19), have spread from China to the rest of the world $[2,3]$.

Currently the percentage of asymptomatic infected carriers is unknown, but different studies indicate that this percentage could be very high [4]. In symptomatic patients, the clinical spectrum of SARS-CoV-2 infection appears to be wide, encompassing mild upper respiratory tract illness, and severe viral pneumonia with respiratory failure and even death [5]. Among patients with confirmed SARS-CoV-2 infection, we encountered a significant number of patients with acute renal dysfunction. Some even progressed to renal failure and required dialysis. One of the main complications that are being observed in these patients is the rapid clinical deterioration that some people exhibit. In this sense, early estimation of risk factors for severe disease and death in these patients seems to be a priority. In previous reports of SARS and MERS-CoV infections, acute kidney injury (AKI) developed in 5-15\% cases and carried a high (60-90\%) mortality [6]. Nevertheless, the incidence and prognosis of acute kidney failure in SARS-CoV-2 patients is unknown and information about the clinical impact remains sparse.

Here, we present details of an international registry of patients discharged from a hospital with laboratoryconfirmed or high suspicion SARS-CoV-2 infection and definite clinical outcomes. The clinical effects of renal function in SARS-CoV-2 infection were explored.

\section{Methods}

\section{Study design and population}

Renal function was recorded on admission from a cohort study of 758 patients with confirmed or highly suspected COVID-19 infection included in the multicenter international HOPE Registry (https://www.hopeprojectmd.com), Registry NCT04334291 on ClinicalTrials.gov. The HOPERegistry was established through an international consortium. Detailed information about participating countries and hospitals, protocol and definitions are reported on website of the Registry. In this interim analysis hospital data and patients were included until the second of April 2020. All Patients discharged (deceased or alive) from any hospital center with a confirmed diagnosis or a COVID-19 high suspicion were included in the HOPE Registry. The local ethics committee approved this study and was consistent with the guidelines of Helsinki. A list of participating hospitals, investigators, collaborators and the protocol are available in the appendix.

\section{Outcome definition}

We assessed the impact of kidney failure severity on admission on the prognosis of 758 patients with COVID19. Patients were stratified into three groups according to the estimated glomerular filtration rate (eGFR) on admission: Absence of significant renal failure if GFR $>60 \mathrm{~mL} /$ $\min / 1.73 \mathrm{~m}^{2}$; moderate renal failure if GFR between $30-60 \mathrm{~mL} / \mathrm{min} / 1.73 \mathrm{~m}^{2}$; and severe renal failure if GFR $<30 \mathrm{~mL} / \mathrm{min} / 1.73 \mathrm{~m}^{2}$. Creatinine clearance has been calculated using Chronic Kidney Disease Epidemiology Collaboration (CKD-EPI) formula.

Primary endpoint was defined as all-cause in-hospital death. Secondary outcomes were in-hospital complications such respiratory insufficiency, AKI, pneumonia, sepsis and embolic events. Chronic kidney disease (CKD) was identified based on the patient's medical history. AKI was identified at admission when the patient had no previous CKD and creatinine clearance at admission was $<60 \mathrm{~mL} /$ $\min / 1.73 \mathrm{~m}^{2}$. AKI during admission was diagnosed according the standard definition of AKI in adults: an increase in serum creatinine by $\geq 26 \mu \mathrm{mol} / \mathrm{L}(0.3 \mathrm{mg} / \mathrm{dL})$ within $48 \mathrm{~h}$, or an increase in serum creatinine to $>1.5$ times baseline within the previous 7 days, or urine volume $<0.5 \mathrm{~mL} / \mathrm{kg} / \mathrm{h}$ for $>6 \mathrm{~h}$. Respiratory insufficiency was defined by an arterial oxygen tension $<60 \mathrm{mmHg}$, and/or arterial carbon dioxide tension $>45 \mathrm{mmHg}$ or both, or the presence of $\mathrm{O} 2$ saturation less than $92 \%$, at room air.

\section{Management and treatment}

There is little direct evidence to inform management of COVID-19. Pending the publication of ongoing clinical trials, recently, the American Thoracic Society-led International Task Force has published some recommendations in which the important lack of evidence in this regard can be verified [7]. For this reason, the treatment used by each of the centers involved in this registry may differ, and it is based on individualized protocols according to their selfexperience treating COVID-19 infection and their local hospital resources.

\section{Statistical analysis}

Data is presented as mean \pm standard deviation for continuous variables with a normal distribution, median 
(interquartile range) for continuous variables with a nonnormal distribution, and as frequency (\%) for categorical variables. Student's $t$ test and the Mann-Whitney U-test were used to compare continuous variables with normal and non-normal distributions, when needed. The Chi squaredtest or Fisher's exact test was used to compare categorical variables. Univariate analysis was performed for qualitative variables and reported as odds ratios (OR) with 95\% CI. Given the multiplicity of variables, only factors with $\mathrm{p}<0.01$ on univariate analysis (dislipemia, diabetes mellitus, smoke, chronic kidney failure, heart disease, lung disease, cerebrovascular disease, connective disease, cancer, immunosuppression condition, RAAS-inhibitors treatment, aspirin treatment, anticoagulation treatment, statin treatment, saturation $\mathrm{O} 2<92 \%$ on admission, d-dimer elevation, PCR elevation, lactate dehydrogenase elevation, eGFR on admission) were entered into the Cox multivariate regression analysis to define independent risk factors for the main outcome. Possible collinearity and interactions were evaluated with the introduction of multiplicative terms calculating the tolerance and the variance inflation factor. The relationship between creatinine clearance and the predicted probability of death was graphically represented after modeling this association using fractional polynomials. All tests were two-sided, and a $P$ value less than 0.05 was considered statistically significant. Statistical analysis was performed with the IBM SPSS 20.0 software package and STATA software, version 15.

\section{Results}

\section{Baseline characteristics}

A total of 758 patients were included in our study. The percentage of testing positive patients for SARS-CoV2 infection by Nasopharyngeal PCR was $90.8 \%$. Table 1 shows the baseline characteristics of COVID-19 patients. Mean age was $66 \pm 18$ years, $58.6 \%$ of patient were male and the median duration from illness onset to admission was 6 (IQR 5) days. Of the total reported patients 317 (48.9\%) had hypertension, $290(38.7 \%)$ dyslipidemia, 138 (21.9\%) diabetes mellitus, $149(19.5 \%)$ and $199(26.1 \%)$ had some previous pulmonary or cardiac condition, respectively. Only $8.5 \%$ of patients had a history of CKD, however, close $30 \%$ of patients had any sort of impaired kidney function according to their eGFR upon hospital admission.

Patients were categorized in 3 groups according to eGFR on the admission $\left(e G F R>60 \mathrm{~mL} / \mathrm{min} / 1.73 \mathrm{~m}^{2}[\mathrm{n}=526]\right.$, eGFR 30-60 mL/min/1.73 $\mathrm{m}^{2}[\mathrm{n}=177]$ and eGFR $<30 \mathrm{~mL} /$ $\left.\min / 1.73 \mathrm{~m}^{2}[\mathrm{n}=55]\right)$. When we compared these groups (Table 1), we observed that patients with renal injury (eGFR 30-60 $\mathrm{mL} / \mathrm{min} / 1.73 \mathrm{~m}^{2}$ and eGFR $<30 \mathrm{~mL} / \mathrm{min} / 1.73 \mathrm{~m}^{2}$ groups vs eGFR $>60 \mathrm{~mL} / \mathrm{min} / 1.73 \mathrm{~m}^{2}$ group) were older and presented a greater number of comorbidities. Furthermore, these groups had more frequently received prior treatment with antiplatelets, anticoagulants and renin-angiotensin-aldosterone system (RAAS) inhibitors. Groups with poorer eGFR on admission had a higher proportion of CKD.

\section{Comparison of clinical aspects on admission between different groups}

Table 1 shows the comparison of signs, symptoms and laboratory test on admission between three groups. In general, patients with poorer kidney function (eGFR 30-60 mL/ $\mathrm{min} / 1.73 \mathrm{~m}^{2}$ and eGFR $<30 \mathrm{~mL} / \mathrm{min} / 1.73 \mathrm{~m}^{2}$ groups vs eGFR $>60 \mathrm{~mL} / \mathrm{min} / 1.73 \mathrm{~m}^{2}$ group) went to the hospital sooner after the symptoms onset and they were in a worse clinical situation. Fever was the most frequent reason for seeking medical attention. We observed that groups with poorer renal function (eGFR $30-60 \mathrm{~mL} / \mathrm{min} / 1.73 \mathrm{~m}^{2}$ and eGFR $<30 \mathrm{~mL} / \mathrm{min} / 1.73 \mathrm{~m}^{2}$ groups) had a lower incidence of general symptoms (cough, anosmia, dysgeusia, myalgia or arthralgia); albeit, respiratory failure was more frequent. Laboratory parameters on admission suggestive of systemic inflammatory response (SIRS) or coagulopathies, such as D-dimer or lymphopenia, were also more frequent in patients with worse eGFR (eGFR 30-60 mL/min/1.73 $\mathrm{m}^{2}$ and $\mathrm{eGFR}<30 \mathrm{~mL} / \mathrm{min} / 1.73 \mathrm{~m}^{2}$ groups).

\section{Treatment and outcomes during admission}

Management is depicted in Table 1. The specific drug most frequently used was hydroxychloroquine $(79.7 \%)$, followed by antibiotics $(70.7 \%)$ and antiviral drug, (lopinavir/ritonavir) (68.7\%). Corticoids were prescribed in approximately $20 \%$ of the cases. Treatment with RAAS inhibitors was maintained in $14 \%$ of patients during admission. In the respiratory support sphere, prone was used in $10 \%$, and non-invasive mechanical ventilation in $18 \%$. An invasive mechanical ventilation approach was required in more than $5 \%$. When we compared these groups according to eGFR, groups with poorer renal function on admission were less frequently treated with hydroxychloroquine (eGFR $>60$ vs eGFR $30-60$ vs and eGFR $<30,82.3 \%$ vs $76.5 \%$ vs $63.5 \%$, $\mathrm{p}<0.001)$ and antivirals (Lopinavir/Ritonavir) (eGFR $>60$ vs eGFR $30-60$ vs and eGFR $<30,71.3 \%$ vs $63.9 \%$ vs $58.0 \%, \mathrm{p}<0.001)$.

Regarding in-hospital events, the most common was bilateral pneumonia, reported in more than $75 \%$ of the cases, with associated respiratory insufficiency in $43.4 \%$. Renal failure and sepsis or SIRS were common, in more than $15 \%$. Sepsis and respiratory failure were more frequent in groups with eGFR $<60 \mathrm{ml} / \mathrm{min} / \mathrm{m}^{2}$ on admission. The incidence of AKI at or during admission in our series was $19.7 \%$. When we analyze the development of AKI during admission, we 
Table 1 Baseline characteristics, signs, symptoms, laboratory test, treatments, complications and outcomes of different groups according to the glomerular filtration rate

\begin{tabular}{|c|c|c|c|c|}
\hline & $\begin{array}{l}\mathrm{eGFR}>60 \mathrm{~mL} / \mathrm{min} / 1.73 \\
\mathrm{~m}^{2}(\mathrm{~N}=526)\end{array}$ & $\begin{array}{l}\text { eGFR } 30-60 \mathrm{~mL} / \mathrm{min} / 1.73 \\
\mathrm{~m}^{2}(\mathrm{~N}=177)\end{array}$ & $\begin{array}{l}\mathrm{eGFR}<30 \mathrm{~mL} / \mathrm{min} / 1.73 \\
\mathrm{~m}^{2}(\mathrm{~N}=55)\end{array}$ & $\mathrm{p}$ \\
\hline Female (\%) & $219(41.6 \%)$ & $68(38.4 \%)$ & $26(47.3 \%)$ & 0.487 \\
\hline Age (years) & $61 \pm 17$ & $78 \pm 11$ & $79 \pm 13$ & $<0.001$ \\
\hline Hypertension & $192(36.6 \%)$ & $132(75.4 \%)$ & $44(80.0 \%)$ & $<0.001$ \\
\hline Dyslipidemia & $164(31.7 \%)$ & $99(57.6 \%)$ & $25(45.5 \%)$ & $<0.001$ \\
\hline Diabetes & $74(14.6 \%)$ & $42(25.0 \%)$ & $20(37.7 \%)$ & $<0.001$ \\
\hline Smoker & $96(20.6 \%)$ & $53(34.2 \%)$ & $15(30.6 \%)$ & 0.002 \\
\hline Lung disease & $91(17.3 \%)$ & $42(23.7 \%)$ & $15(27.3 \%)$ & 0.056 \\
\hline Known chronic renal disease & $6(1.2 \%)$ & $23(13.9 \%)$ & $30(58.8 \%)$ & $<0.001$ \\
\hline Obesity & $85(16.1 \%)$ & $31(17.5 \%)$ & $16(29.0 \%)$ & 0.005 \\
\hline Cardiovascular disease & $88(16.7 \%)$ & $84(47.5 \%)$ & $26(47.3 \%)$ & $<0.001$ \\
\hline Cerebrovascular disease & $32(6.4 \%)$ & $38(23.0 \%)$ & $10(18.5 \%)$ & $<0.001$ \\
\hline Any cancer & $64(12.2 \%)$ & $32(18.1 \%)$ & $11(20.0 \%)$ & 0.064 \\
\hline Previous statin treatment & $37(8.9 \%)$ & $30(25.4 \%)$ & $6(16.7 \%)$ & 0.006 \\
\hline Previous antiaggregant treatment & $59(11.6 \%)$ & $52(31.0 \%)$ & $15(27.8 \%)$ & $<0.001$ \\
\hline Previous anticoagulant treatment & $44(8.8 \%)$ & $43(25.3 \%)$ & $12(23.1 \%)$ & $<0.001$ \\
\hline Previous RAAS inhibitors treatment & $141(27.4 \%)$ & $109(63.7 \%)$ & $33(61.1 \%)$ & $<0.001$ \\
\hline Asymptomatic & $9(1.7 \%)$ & $7(4.0 \%)$ & $1(1.9 \%)$ & 0.226 \\
\hline Shortness of breath & $294(55.8 \%)$ & $106(59.8 \%)$ & $37(67.2 \%)$ & 0.003 \\
\hline Anosmia & $22(5.0 \%)$ & $1(0.7 \%)$ & $2(2.1 \%)$ & 0.011 \\
\hline Dysgeusia & $24(5.4 \%)$ & $0(0.0 \%)$ & $1(1.8 \%)$ & 0.004 \\
\hline Sorethroat & $43(9.5 \%)$ & $14(9.0 \%)$ & $3(6.7 \%)$ & 0.820 \\
\hline Fever $\left(>38.2^{\circ} \mathrm{C}\right)$ & $434(84.4 \%)$ & $138(78.9 \%)$ & $38(70.4 \%)$ & 0.016 \\
\hline Cough & $373(73.0 \%)$ & $110(63.6 \%)$ & $27(52.9 \%)$ & 0.002 \\
\hline Diarrhea & $81(17.2 \%)$ & $15(9.4 \%)$ & $9(19.1 \%)$ & 0.051 \\
\hline Myalgias or arthralgias & $161(33.6 \%)$ & $43(25.9 \%)$ & $6(12.2 \%)$ & 0.003 \\
\hline Tachypnea & $87(19.0 \%)$ & $50(28.1 \%)$ & $26(39.9 \%)$ & $<0.001$ \\
\hline Saturation on admission $<92 \%$ & $153(30.5 \%)$ & $104(59.8 \%)$ & $28(52.8 \%)$ & $<0.001$ \\
\hline D-Dimer elevation & $260(61.0 \%)$ & $115(79.9 \%)$ & $25(65.8 \%)$ & $<0.001$ \\
\hline Procalcitonin elevation & $75(19.5 \%)$ & $36(26.5 \%)$ & $19(48.7 \%)$ & $<0.001$ \\
\hline PCR elevation & $463(90.6 \%)$ & $165(95.4 \%)$ & $53(96.4 \%)$ & 0.063 \\
\hline Transaminases elevation & $185(41.8 \%)$ & $67(42.9 \%)$ & $20(41.7 \%)$ & 0.966 \\
\hline Ferritin elevation & $160(56.9 \%)$ & $64(60.4 \%)$ & $19(67.9 \%)$ & 0.485 \\
\hline Lactate dehydrogenase elevation & $310(71.8 \%)$ & $107(78.7 \%)$ & $33(73.3 \%)$ & 0.281 \\
\hline Onset creatinine levels (mg/dL) & $0.8 \pm 0.2$ & $1.4 \pm 0.3$ & $3.2 \pm 2.0$ & $<0.001$ \\
\hline Total onset lymphocytes count (/UL) & $1261 \pm 1390$ & $1202 \pm 2130$ & $870 \pm 514$ & 0.264 \\
\hline Duration from illness onset to first admission & $6.5 \pm 6.0$ & $4.9 \pm 4.1$ & $4.4 \pm 3.2$ & 0.001 \\
\hline Acute kidney injury & $33(6.7 \%)$ & $72(43.4 \%)$ & $44(86.3 \%)$ & $<0.001$ \\
\hline Bilateral pneumonia & $349(66.3 \%)$ & $127(71.8 \%)$ & $36(40.8 \%)$ & 0.311 \\
\hline Sepsis & $57(11.9 \%)$ & $42(26.4 \%)$ & $20(40.8 \%)$ & $<0.001$ \\
\hline Ischemic event & $2(0.4 \%)$ & $2(1.3 \%)$ & $0(0.0 \%)$ & 0.438 \\
\hline Respiratory insufficiency & $176(35.4 \%)$ & $122(72.2 \%)$ & $31(62.0 \%)$ & $<0.001$ \\
\hline High Flow Nasal Cannula & $120(24.1 \%)$ & $63(37.3 \%)$ & $25(48.1 \%)$ & $<0.001$ \\
\hline Non-invasive mechanical ventilation & $80(16.1 \%)$ & $44(26.2 \%)$ & $11(20.4 \%)$ & 0.015 \\
\hline Invasive mechanical ventilation & $19(4.0 \%)$ & $12(7.5 \%)$ & $2(4.2 \%)$ & 0.198 \\
\hline Use of corticoids & $79(16.3 \%)$ & $48(29.3 \%)$ & $6(11.8 \%)$ & $<0.001$ \\
\hline Use of hydroxychloroquine & $408(82.3 \%)$ & $130(76.5 \%)$ & $33(63.5 \%)$ & 0.003 \\
\hline Use of antiviral drugs & $357(71.3 \%)$ & $108(63.9 \%)$ & $29(58.0 \%)$ & 0.050 \\
\hline Use of interferon or similar & $66(13.7 \%)$ & $27(16.4 \%)$ & $6(12.2 \%)$ & 0.642 \\
\hline
\end{tabular}


Table 1 (continued)

\begin{tabular}{|c|c|c|c|c|}
\hline & $\begin{array}{l}\mathrm{eGFR}>60 \mathrm{~mL} / \mathrm{min} / 1.73 \\
\mathrm{~m}^{2}(\mathrm{~N}=526)\end{array}$ & $\begin{array}{l}\text { eGFR } 30-60 \mathrm{~mL} / \mathrm{min} / 1.73 \\
\mathrm{~m}^{2}(\mathrm{~N}=177)\end{array}$ & $\begin{array}{l}\mathrm{eGFR}<30 \mathrm{~mL} / \mathrm{min} / 1.73 \\
\mathrm{~m}^{2}(\mathrm{~N}=55)\end{array}$ & $\mathrm{p}$ \\
\hline Use of tocilizumab or similar & $26(5.4 \%)$ & $15(9.2 \%)$ & $1(2.0 \%)$ & 0.099 \\
\hline Use of antibiotics & $346(68.8 \%)$ & $127(74.7 \%)$ & $40(76.9 \%)$ & 0.204 \\
\hline ACEI ARBS during in hospital stay & $57(12.4 \%)$ & $28(19.3 \%)$ & $10(22.2 \%)$ & 0.038 \\
\hline Hospitalization stay (days) & $6.5 \pm 4.3$ & $6.7 \pm 4.8$ & $5.7 \pm 4.8$ & 0.395 \\
\hline
\end{tabular}

$e G F R$ estimated glomerular filtration rate

observe that a $5.2 \%$ of the patients in eGFR $>60$ group presented a worsening of their renal function vs $31.8 \%$ in eGFR 30-60 group vs $56.0 \%$ in eGFR $<30$ group, $\mathrm{p}<0.001$.

\section{Mortality risk assessment}

Kaplan-Meier survival landmark analysis according to the glomerular filtration rate are shown in Fig. 1, logrank test global $\mathrm{p}<0.001$ and log-rank test between each group. $\mathrm{p}<0.001$. We investigated those factors associated with a higher risk of suffering from the primary outcome defined as in-hospital mortality. Univariate regression analyses are shown in Supplementary Table 1. After univariate analyses, we performed a Cox multivariate regression analysis to identify those variables that were independently associated with a greater risk of in-hospital mortality. Most relevant risk-factors on admission for inhospital death (Table 2) in the logistic regression model were: age, hypertension, kidney function, the presence of $\mathrm{O} 2$ saturation less than $92 \%$ and an elevated lactate dehydrogenase. Renal function at admission behaved as an independent prognostic factor when it was categorically evaluated (eGFR groups) and when it was assessed as a continuous variable (creatinine value at admission), Table S2. The impact of creatinine clearance on mortality is displayed graphically in Fig. 2. As creatinine clearance decreased, the probability of death increased. Importantly, when we excluded patients with known chronic

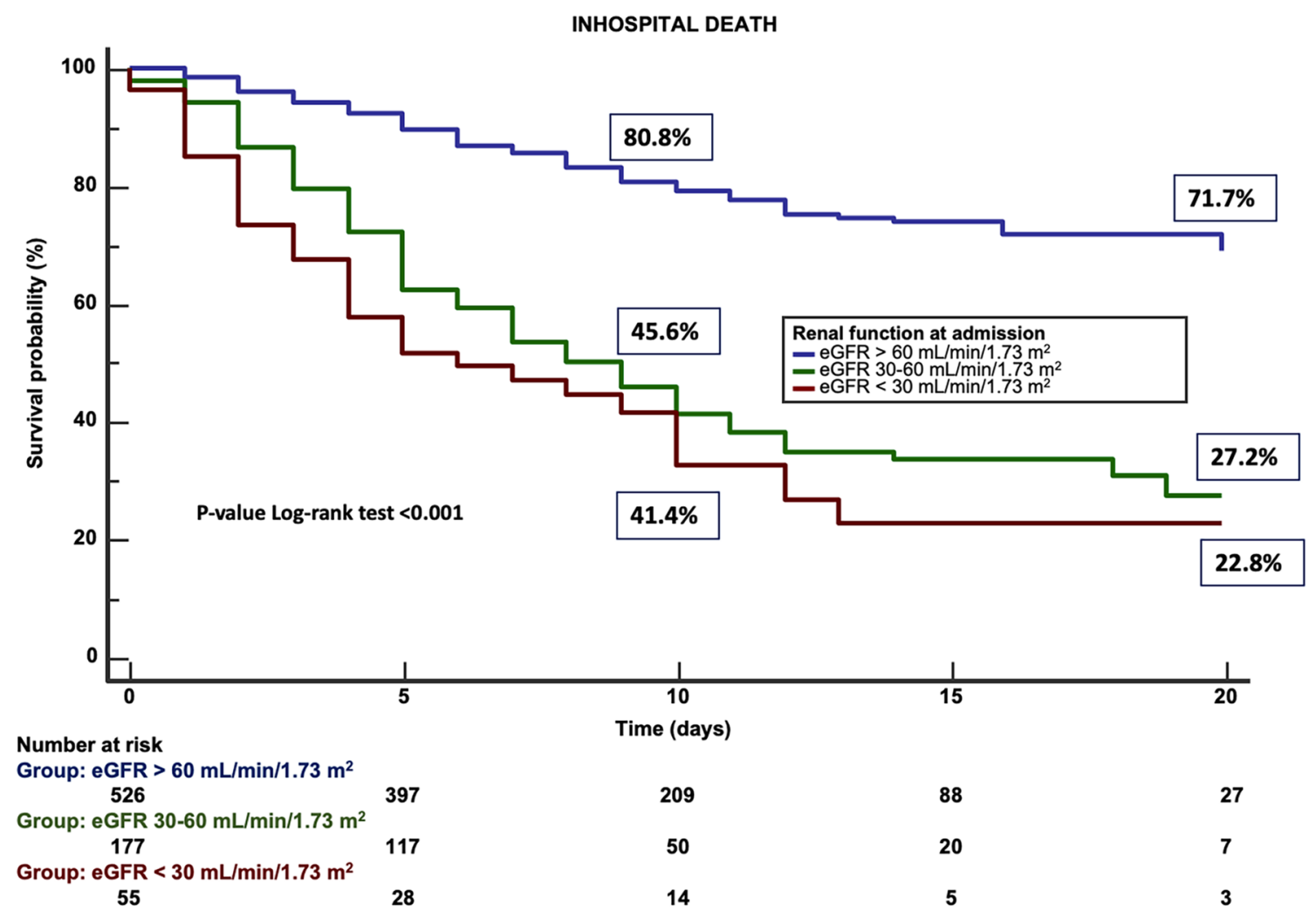

Fig. 1 Kaplan-Meier survival landmark analysis according to the glomerular filtration rate 
Table 2 Cox multivariate regression analysis regarding risk factors on admission associated with in-hospital death

\begin{tabular}{llr}
\hline & HR $($ CI 95\%) & \multicolumn{1}{c}{$\mathrm{p}$} \\
\hline Hypertension & $1.642(1.105-2.179)$ & 0.038 \\
Age & $1.034(1.021-1.048)$ & $<0.001$ \\
Saturation on admission $<92 \%$ & $3.310(2.362-4.369)$ & $<0.001$ \\
Lactate dehydrogenase elevation & $1.768(1.161-2.690)$ & 0.008 \\
eGFR 30-60 mL/min/1.73 m ${ }^{2 a}$ & $2.205(1.573-3.091)$ & $<0.001$ \\
eGFR $<30 \mathrm{~mL} / \mathrm{min} / 1.73 \mathrm{~m}^{2 \mathrm{a}}$ & $4.925(2.152-5.244)$ & $<0.001$ \\
\hline
\end{tabular}

RASS renin-angiotensin-aldosterone system

${ }^{a}$ Reference to eGFR $>60 \mathrm{~mL} / \mathrm{min} / 1.73 \mathrm{~m}^{2}$ group

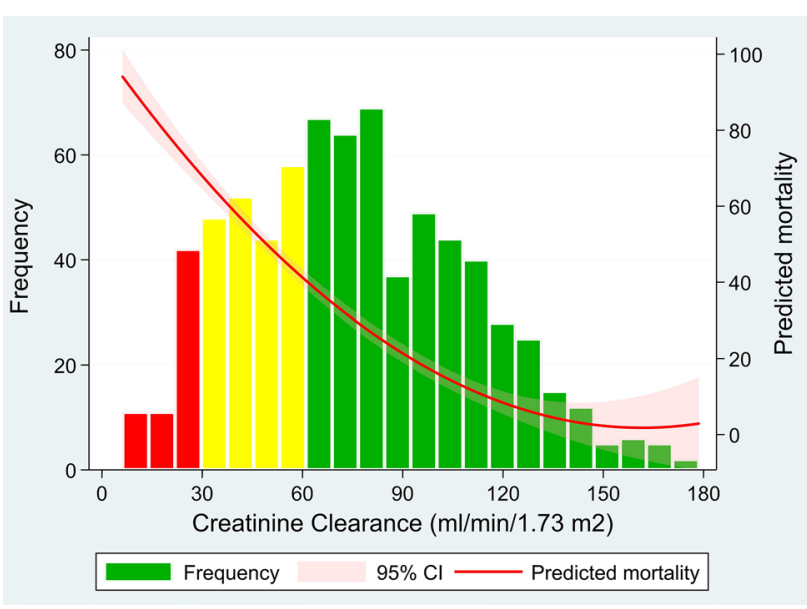

Fig. 2 Association between creatinine clearance and predicted mortality. The predicted probability of all-cause death (red line) is shown, together with $95 \%$ confidence interval, after adjustment for age, hypertension, diabetes, dislipemia, smoking habit, any heart disease, any lung disease, any cerebrovascular disease, any immunosuppression condition, RAAS inhibitors treatment, aspirin treatment, anticoagulation treatment, statin treatment, saturation $\mathrm{O} 2<92 \%$ on admission, D-dimer elevation, PCR elevation, and lactate dehydrogenase elevation at admission. The $\mathrm{x}$-axis shows the values of creatinine clearance as continuous variable. Histograms show the population distribution according to creatinine clearance levels (color figure online)

renal failure from the analysis the results remained consistent (Table S3).

Because hypertension can act as a confounding factor, a new logistic regression analysis was performed excluding hypertensive patients. The deterioration of renal function continued to be independently associated with a worse prognosis in these group of patients $(\mathrm{OR}=8.6,95 \%$ CI $1.8-40.5, \mathrm{p}<0.001$ in eGFR $<30 \mathrm{~mL} /$ $\mathrm{min} / 1.73 \mathrm{~m}^{2}$ group; $\mathrm{OR}=2.2,95 \% \mathrm{CI} 0.8-5.9, \mathrm{p}=0.128$ in
eGFR 30-60 mL/min $/ 1.73 \mathrm{~m}^{2}$ group; reference $>60 \mathrm{~mL} /$ $\mathrm{min} / 1.73 \mathrm{~m}^{2}$ group).

\section{Discussion}

In this large international registry conducted in Europe and America, we observed a high prevalence of kidney disease in hospitalized COVID-19 patients. Close to 30\% of them had evidence of kidney disease on admission, with elevated serum creatinine, and this was associated with greater in-hospital mortality. Two factors may have been involved in this. On the one hand, increased susceptibility to infection in patients with CKD, and on the other hand, the acute deterioration of renal function related to COVID19. One possible explanation of the high prevalence of kidney involvement at hospital admission is that some of the patients with COVID-19 had a past history of CKD. Such patients have a proinflammatory state with functional defects in innate and adaptive immune cell populations [8] and are known to have a higher risk pneumonia [9]. Interestingly, in our registry only $8.5 \%$ had a history of CKD, while roughly $35 \%$ of all the patients displayed any sort of kidney function deterioration on admission; Hence, there must be a direct causal relation-ship between AKI and COVID-19.

To understand why kidney failure may be a crucial factor in the evolution of patients with SARS-CoV-2 infection, it is important to know the evolution of COVID19. Acute disease progression can be divided into three distinct phases: an early infection phase, a pulmonary phase, and a severe hyperinflammation phase [10-12]. The greatest susceptibility to infection would focus on this first phase of early infection, during which, the virus infiltrates the lung parenchyma and begins to proliferate. In this phase of infection, the RAAS seems to play a key role. SARS-CoV-2 interacts with the RAAS through angiotensin-converting enzyme 2 (ACE2), an enzyme that physiologically counters RAAS activation but also functions as a receptor for SARS viruses [13]. ACE2 is a type I membrane protein expressed in lung, heart, kidney, and intestine but mainly associated with cardiovascular diseases [13]. Recent human tissue RNA-sequencing data demonstrated that ACE2 expression in the kidneys was nearly 100-fold higher than in the lung [14]. Therefore, kidney disease may be caused by coronavirus entering kidney cells through an ACE2-dependent pathway. Moreover, RAAS activity is clearly increased in patients with $\mathrm{CKD}$, so there is a systemic increase in ACE2 receptors that might be translated into an easier SARS-CoV-2 cell infection.

Another factor that may explain a worse prognosis in patients with kidney damage is that the novel coronavirus 
may exert direct cytopathic effects on kidney tissue. This is supported by the detection of polymerase chain reaction fragments of coronavirus blood and urine in patients with the 2003 SARS and COVID-19 viral infections [15-17]. A recent in vitro study concluded that the cytopathic effects of SARS-CoV-2 on podocytes and proximal straight tubule cells may cause AKI in patients with COVID-19, especially in Occidental populations due to the highest expression of the ACE2 receptor in these cells [18]. Recently, Li et al. observed renal structural anomalies by CT scan in $100 \%$ of patients infected with SARSCoV-2 [14], and this direct virus-induced damage has been observed in kidney biopsies performed on patients with COVID-19 [19].

Another proposed mechanism that justifies kidney injury during SARS-CoV-2 infection is cytokine-mediated damage. A non-negligible percentage of patients will develop during the inflammatory phase of the disease an exuberant response, called cytokine storm, resulting in uncontrolled pulmonary inflammation, likely a leading cause of case fatality [13]. Rapid viral replication and cellular damage, viral-mediated ACE2 downregulation and shedding, and antibody dependent enhancement are responsible for aggressive inflammation caused by SARS-CoV-2. The initial onset of rapid viral replication may cause massive epithelial and endothelial cell death and vascular leakage, triggering the production of pro-inflammatory cytokines and chemokines that can cause direct kidney damage through apoptosis of the renal tubular epithelial cells [20]. In clinical practice, an altered kidney function should be given particular attention, as an impaired renal function could be an early marker of a hyperinflammatory phase in a patient who has not yet developed severe respiratory failure, therefore, monitoring kidney function must be emphasized even in patients with mild respiratory symptoms. Early detection of renal abnormalities could involve the early start of drugs against the inflammatory cascade and could help to improve the vital prognosis of COVID-19. Furthermore, it could allow a better treatment adjustment because many of the drugs that are being used for the treatment of COVID-19 are nephrotoxic through pharmacological/ iatrogenic mechanisms.

Several publications have associated hypertension with a higher probability of infection and a worse evolution in patients with COVID-19 [21, 22], and our data goes in the same direction. Although hypertension is a highly prevalent cardiovascular risk factor in CKD patients, our data supports that renal failure continues to be an independent prognostic factor irrespective of the presence or absence of concomitant hypertension.

Our findings warn of the possibility that patients with chronic renal failure could be patients at risk for
COVID-19 infection. In this sense, recent publications attempt to protocolize the care of CKD patients to reduce the risk of infection [23-25].

\section{Limitations}

We need to consider the constraints of a study of this design. It is possible that some incident events in the participating centers have not been diagnosed and/or reported. The calculation of the incidence of the events is not precise since the recruitment was performed in participating centers without other sampling procedure than the broad inclusion criteria (hospital discharge) and would vary depending on the patient's, hospital, country or local pandemic curve. Regarding the management applied, at all times they were decided by the attending medical team, as well as in the comparison group. The high incidence of respiratory failure could be related to the definition used.

Other considerations to take into account are: an accurate baseline serum creatinine was not available, which may have led to an underestimation of AKI or erroneous associations; although we attempted to adjust for many confounders, other unmeasured or unknown confounders might have played a role; despite we tried to report all the treatments used during admission, the protocols between the centers were different, which may influence the results. The precise impact of COVID-19 on kidney structure and function and the incidence of CKD in these patients warrant further investigation.

\section{Conclusions}

Our findings show the prevalence of AKI on admission in patients with COVID-19 is high and is associated with a greater in-hospital mortality rate. Physicians should closely monitor any patient with impaired renal function on admission, regardless of respiratory status. Our data comes from a multicenter registry and therefore does not allow to have a precise mortality risk assessment. More studies are needed to confirm these findings.

Acknowledgements Cardiovascular Excellence SL, for their essential support in the database and HOPE webpage. All HOPE researchers.

Funding Non conditioned Grant (FUNDACIÓN INTERHOSPITALARIA PARA LA INVESTIGACIÓN CARDIOVASCULAR, FIC. Madrid, Spain). This nonprofit institution had no role in the study design; in the collection, analysis, interpretation of data; in the writing of the report; nor in the decision to submit the paper for publication.

\section{Compliance with ethical standards}

Conflict of interest The authors declare that they have no conflict of interest. 
Ethical approval The study was approved by the Ethics Research Committee from Hospital Clinico San Carlos (Madrid, Spain) (20/241-E) and the Spanish Drug Agency authorities (AEMPS classification: EPAOD) and by local committees when needed.

Informed consent Written informed consent was waived owing to the severity of the situation and the use of deidentified retrospective data. However, verbal authorization from either patients or caregivers was required.

\section{References}

1. WHO (2020) Novel coronavirus-China. http://www.who.int/csr/ don/12-january-2020-novel-coronavirus-china/en/. Accessed 24 Apr 2020

2. Center for Systems Science and Engineering. Coronavirus COVID-19 global cases. https://www.gisanddata.maps.arcgi s.com/apps/opsdashboard/index.html\#/bda7594740fd40299423 467b48e9ecf6. Accessed 20 Apr 2020

3. Chan JF, Yuan S, Kok KH et al (2020) A familial cluster of pneumonia associated with the 2019 novel coronavirus indicating person-to-person transmission: a study of a family cluster. Lancet 395(10223):514-523. https://doi.org/10.1016/S0140 -6736(20)30154-9

4. Flaxman S, Mishra S, Gandy A et al (2020) Report 13: estimating the number of infections and the impact of nonpharmaceutical interventions on COVID-19 in 11 European countries. Imperial College, London. https://doi.org/10.25561/77731

5. Zhou F, Yu T, Du R et al (2020) Clinical course and risk factors for mortality of adult inpatients with COVID-19 in Wuhan, China: a retrospective cohort study. Lancet 395(10229):1054-1062. https ://doi.org/10.1016/S0140-6736(20)30566-3

6. Chu KH, Tsang WK, Tang CS et al (2005) Acute renal impairment in coronavirus-associated severe acute respiratory syndrome. Kidney Int 67(2):698-705

7. Wilson KC, Chotirmall SH, Bai C, Rello J (2020) COVID-19: interim guidance on management pending empirical evidence. https://www.thoracic.org/covid/covid-19-guidance.pdf. Accessed 20 Apr 2020

8. Betjes MGH (2013) Immune cell dysfunction and inflammation in end-stage renal disease. Nat Rev Nephrol 9(5):255-265. https ://doi.org/10.1038/nrneph.2013.44

9. Sibbel S, Sato R, Hunt A, Turenne W, Brunelli SM (2016) The clinical and economic burden of pneumonia in patients enrolled in Medicare receiving dialysis: a retrospective, observational cohort study. BMC Nephrol 17(1):199. https://doi.org/10.1186/s1288 2-016-0412-6

10. Fu Y, Cheng Y, Wu Y (2020) Understanding SARS-CoV-2-mediated inflammatory responses: from mechanisms to potential therapeutic tools. Virol Sin. https://doi.org/10.1007/s12250-020-00207 -4 (Epub ahead of print)

11. Siddiqi HK, Mehra MR (2020) COVID-19 illness in native and immunosuppressed states: a clinical-therapeutic staging proposal. J Heart Lung Transplant. https://doi.org/10.1016/j.healu n.2020.03.012

12. Channappanavar R, Perlman S (2017) Pathogenic human coronavirus infections: causes and consequences of cytokine storm and immunopathology. Semin Immunopathol 39(5):529-539. https:// doi.org/10.1007/s00281-017-0629-x

13. Jin Y, Yang H, Ji W et al (2020) Virology, epidemiology, pathogenesis, and control of COVID-19. Viruses 12(4):372. https://doi. org/10.3390/v12040372

14. Anti-2019-nCoV Volunteers, Li Z, Wu M et al (2020) Caution on kidney dysfunctions of COVID-19 patients. medRxiv. https://doi. org/10.1101/2020.02.08.20021212

15. Peiris JS, Chu CM, Cheng VC et al (2003) Clinical progression and viral load in a community outbreak of coronavirus-associated SARS pneumonia: a prospective study. Lancet 361(9371):17671772. https://doi.org/10.1016/S0140-6736(03)13412-5

16. Huang C, Wang Y, Li X et al (2020) Clinical features of patients infected with 2019 novel coronavirus in Wuhan, China. Lancet 395(10223):497-506. https://doi.org/10.1016/S0140 $-6736(20) 30183-5$

17. Cha RH, Yang SH, Moon KC et al (2016) A case report of a middle east respiratory syndrome survivor with kidney biopsy results. J Korean Med Sci 31(4):635-640. https://doi.org/10.3346/ jkms.2016.31.4.635

18. Pan X-W, Da Xu, Zhang H, Zhou W, Wang L-H, Cui X-G (2020) Identification of a potential mechanism of acute kidney injury during the COVID-19 outbreak: a study based on single-cell transcriptome analysis. Intensive Care Med. https://doi.org/10.1007/ s00134-020-06026-1

19. Su H, Yang M, Wan C et al (2020) Renal histopathological analysis of 26 postmortem findings of patients with COVID-19 in China. Kidney Int. https://doi.org/10.1016/j.kint.2020.04.003

20. Tisoncik JR, Korth MJ, Simmons CP, Farrar J, Martin TR, Katze MG (2012) Into the eye of the cytokine storm. Microbiol Mol Biol Rev 76(1):16-32. https://doi.org/10.1128/MMBR.05015-11

21. Wang D, Hu B, Hu C et al (2020) Clinical characteristics of 138 hospitalized patients with 2019 novel coronavirus-infected pneumonia in Wuhan, China. JAMA 323(11):1061-1069. https://doi. org/10.1001/jama.2020.1585

22. Guan WJ, Ni ZY, Hu Y et al (2020) Clinical characteristics of coronavirus disease 2019 in China. N Engl J Med. https://doi. org/10.1056/NEJMoa2002032

23. Rombolà G, Brunini F (2020) COVID-19 and dialysis: why we should be worried. J Nephrol 33:401-403. https://doi.org/10.1007/ s40620-020-00737-w

24. Rombolà G, Heidempergher M, Pedrini L et al (2020) Practical indications for the prevention and management of SARS-CoV-2 in ambulatory dialysis patients: lessons from the first phase of the epidemics in Lombardy. J Nephrol 33:193-196. https://doi. org/10.1007/s40620-020-00729-w

25. Manganaro M, Baldovino S (2020) Working group of the piedmont and Aosta Valley section of the SIN. First considerations on the SARS-CoV-2 epidemic in the Dialysis Units of Piedmont and Aosta Valley, Northern Italy. J Nephrol 33(3):393-395. https ://doi.org/10.1007/s40620-020-00732-1

Publisher's Note Springer Nature remains neutral with regard to jurisdictional claims in published maps and institutional affiliations. 


\section{Affiliations}

\section{Aitor Uribarri ${ }^{1}$ - Iván J. Núñez-Gil ${ }^{2}$ - Alvaro Aparisi ${ }^{1}$ - Victor M. Becerra-Muñoz ${ }^{3}$. Gisela Feltes ${ }^{4}$. Daniela Trabattoni ${ }^{5}$. Inmaculada Fernández-Rozas ${ }^{6} \cdot$ María C. Viana-Llamas $^{7} \cdot$ Martino Pepe $^{8}$. Enrico Cerrato ${ }^{9}$. Thamar Capel-Astrua $^{10}$ - Rodolfo Romero ${ }^{11}$ - Alex F. Castro-Mejía ${ }^{12}$ - Ibrahim El-Battrawy ${ }^{13,14}$. Javier López-País ${ }^{15}$. Fabrizio D'Ascenzo ${ }^{16}$. Oscar Fabregat-Andres ${ }^{17}$. Alfredo Bardaji ${ }^{18}$. Sergio Raposeiras-Roubin ${ }^{19} \cdot$ Francisco Marín $^{20}$. Antonio Fernández-Ortiz ${ }^{2}$. Carlos Macaya ${ }^{2}$. Vicente Estrada ${ }^{2}$ on behalf of HOPE COVID-19 Investigators}

1 Instituto de Ciencias del Corazón (ICICOR), CIBER-CV, Hospital Clínico Universitario de Valladolid, C/Ramón y Cajal 3, 47005 Valladolid, Spain

2 Hospital Clínico San Carlos, Universidad Complutense de Madrid, Instituto de Investigación Sanitaria del Hospital Clínico San Carlos (IdISSC), Madrid, Spain

3 Unidad de Gestión Clínica Área del Corazón, Instituto de Investigación Biomédica de Málaga (IBIMA), Hospital Universitario Virgen de la Victoria, Universidad de Málaga (UMA), Centro de Investigación Biomédica en Red de Enfermedades Cardiovasculares (CIBERCV), Málaga, Spain

4 Hospital Nuestra Señora de América, Madrid, Spain

5 Centro Cardiologico Monzino, IRCCS, Milan, Italy

6 Hospital Severo Ochoa, Leganés, Spain

7 Hospital Universitario Guadalajara, Guadalajara, Spain

8 Azienda Ospedaliero-Universitaria Consorziale Policlinico di Bari, Bari, Italy

9 San Luigi Gonzaga University Hospital, Rivoli, Turin, Italy Hospital Virgen del Mar, Madrid, Spain

11 Hospital Universitario Getafe, Madrid, Spain
12 Hospital General del norte de Guayaquil IESS Los Ceibos, Guayaquil, Ecuador

13 First Department of Medicine, Medical Faculty Mannheim, University Heidelberg, Mannheim 68167, Germany

14 DZHK (German Center for Cardiovascular Research), Partner Site, Heidelberg-Mannheim, Mannheim, Germany

15 Complejo Hospitalario Universitario de Santiago de Compostela Santiago de Compostela, Santiago de Compostela, Spain

16 San Giovanni Battista, Turin, Italy

17 Hospital IMED, Valencia, Spain

18 University Hospital Joan XXIII, Tarragona, Spain

19 University Hospital Álvaro Cunqueiro, Vigo, Spain

20 Hospital Clínico Universitario Virgen de la Arrixaca, IMIB-Arrixaca, Universidad de Murcia, CIBERCV, Murcia, Spain 